\title{
Optimization of the Depth Resolution for Deuterium Depth Profiling up to Large Depths
}

\author{
B. Wielunska, M. Mayer, T. Schwarz-Selinger \\ Max-Planck-Institut für Plasmaphysik, Boltzmannstr.2, 85748 Garching, Germany
}

\begin{abstract}
The depth resolution of deuterium depth profiling by the nuclear reaction $\mathrm{D}\left({ }^{3} \mathrm{He}, \mathrm{p}\right) \alpha$ is studied theoretically and experimentally. General kinematic considerations are presented which show that the depth resolution for deuterium depth profiling using the nuclear reaction $\mathrm{D}\left({ }^{3} \mathrm{He}, \mathrm{p}\right) \alpha$ is best at reaction angles of $0^{\circ}$ and $180^{\circ}$ at all incident energies below $9 \mathrm{MeV}$ and for all depths and materials. In order to confirm this theoretical prediction the depth resolution was determined experimentally with a conventional detector at $135^{\circ}$ and an annular detector at $175.9^{\circ}$. Deuterium containing thin films buried under different metal cover layers of aluminium, molybdenum and tungsten with thicknesses in the range of $0.5-11 \mu \mathrm{m}$ served as samples. For all materials and depths an improvement of the depth resolution with the detector at $175.9^{\circ}$ is achieved. For tungsten as cover layer a better depth resolution up to a factor of 18 was determined. Good agreement between the experimental results and the simulations for the depth resolution is demonstrated.
\end{abstract}

\section{Introduction}

The nuclear reaction $\mathrm{D}\left({ }^{3} \mathrm{He}, \mathrm{p}\right) \alpha$ is commonly used to determine the depth profile of deuterium in solids $[1,2,3]$. The total cross section of this reaction has a broad maximum around $630 \mathrm{keV}$ with a cross-section maximum of about $850 \mathrm{mb}$ : This relatively high cross-section allows high detection sensitivity and 
a low detection limit below $100 \mathrm{ppm}$. Differential cross sections for various reaction angles have been measured by a number of authors $[3,4,5]$. Nocente et al. derived a fit formula for the differential cross section, thus allowing to interpolate differential cross-section data for any angle [6]. The reaction has a high Q-value of $18.4 \mathrm{MeV}$ resulting in high energetic protons of $11-14 \mathrm{MeV}$ and $\alpha$ 's of $4.4-7.4 \mathrm{MeV}$ using incident energies of $0.5-6 \mathrm{MeV}$. This high Q-value is advantageous in nuclear reaction analysis as the backscattered ${ }^{3} \mathrm{He}$ particles can be easily stopped by a foil installed in front of the detector. Hence the signal of the high-energetic protons is usually background free.

By utilizing the energy spectrum of the emitted $\alpha$-particles the deuterium depth profile can be derived with a depth resolution of several $\mathrm{nm}$ within the near-surface layer. This requires a special detector geometry with normal incidence and grazing exit angle, resulting in reaction angles close to $100^{\circ}[1]$. The analyzed depth, however, is limited to a near surface layer of several hundred nanometers: For many applications, such as the study of hydrogen isotope diffusion in metals [7] [8] or the determination of the amount of trapped deuterium in wall materials of nuclear fusion experiments [9], this shallow analyzed depth range is largely insufficient.

The deuterium depth profile can be reconstructed up to much larger depths from the proton energy spectra by using multiple incident energies: Analyzed depths up to about $40 \mu \mathrm{m}$ in low-Z materials and up to about $8 \mu \mathrm{m}$ in heavy materials such as tungsten have been demonstrated [2]. Main disadvantage of this method is the limited achievable depth resolution because of angular spread caused by the finite size of the detector aperture and by multiple smallangle scattering in the sample [2]. In [2] it is shown that for low-Z elements geometrical straggling dominates the deterioration of the depth resolution. This problem can be overcome by decreasing the width of the detector aperture (at 
the cost of detector solid angle and thus sensitivity). For samples containing high-Z elements multiple scattering is the dominant process limiting the depth resolution, see [2]: This process cannot be avoided.

For samples containing high-Z elements a possible available optimization parameter with respect to depth resolution is the reaction angle. At many experimental facilities the proton detector is situated at a reaction angle in the vicinity of $135^{\circ}$. This angle is usually used due to the technical limitation that angles around $100^{\circ}$ are blocked by an $\alpha$-detector for high-resolution, near surface D depth profiling and angles in the range $150-170^{\circ}$ are blocked by Rutherford backscattering (RBS) detectors, while reaction angles around $135^{\circ}$ are usually unused and offer enough space for (typically large) proton detectors. This technical solution, however, does not mean that this angle is optimal in any respect and the question arises if there exists a reaction angle which optimizes the depth resolution for deuterium depth profiling up to large depths.

General kinematic considerations for the $\mathrm{D}\left({ }^{3} \mathrm{He}, \mathrm{p}\right) \alpha$ reaction are presented in section 2. It is shown that the optimum depth resolution for this reaction is achieved at reaction angles of $0^{\circ}$ and $180^{\circ}$. These reaction angles are optimal for all materials at all depths and at all incident energies up to at least $9 \mathrm{MeV}$. In order to confirm this theoretical prediction the depth resolutions at $135^{\circ}$ and at $175.9^{\circ}$ were determined experimentally. As sample we used deuterium containing thin films buried under aluminum as low-Z, molybdenum as medium$\mathrm{Z}$ and tungsten as high- $\mathrm{Z}$ materials. The experimental results are presented and compared with simulations in section 3 .

\section{Optimum reaction angle for depth profiling}

To find the best reaction angle for deuterium depth profiling we need to consider the change of the proton energy with respect to the reaction depth. 
The energy $E_{p}$ of the proton after the nuclear reaction is given by the equation for the energy of the light product of an inelastic collision [10]:

$$
E_{p}=B\left[\cos \theta+\sqrt{\left[D / B-\sin ^{2} \theta\right.}\right]^{2}\left(E_{3 H e}+Q\right),
$$

which is valid for $B \leq D$. It was calculated that $B \leq D$ holds for all energies used.

Following abbreviation were used:

$B=\frac{M_{3 H e} M_{p}}{\left(M_{3 H e}+M_{D}\right)\left(M_{p}+M_{\alpha}\right)}\left(E_{3 H e} /\left(E_{3 H e}+Q\right)\right)$,

$D=\frac{M_{D} M_{\alpha}}{\left(M_{3 H e}+M_{D}\right)\left(M_{p}+M_{\alpha}\right)}\left(1+\frac{M_{3 H e} Q}{M_{D}\left(E_{3 H e}+Q\right)}\right)$,

$\theta$ : angle between the incoming beam direction and the exit direction of the proton particle, i.e. the reaction angle.

$\mathrm{M}_{3 H e}, \mathrm{E}_{3 \mathrm{He}}$ : mass, energy of the incident ${ }^{3} \mathrm{He}$ ion.

$\mathrm{M}_{D}$ : mass of the deuterium atom, i.e. the target atom.

$\mathrm{M}_{p}$ : mass of the proton, i.e. the light product of the inelastic collision.

$\mathrm{M}_{\alpha}$ : mass of $\alpha$ particle, i.e. the heavy product of the inelastic collision.

Q: energy released by the reaction.

For further details please refer to [10].

The energy of the proton $\mathrm{E}_{p}$ (eq. 1 ) is illustrated in fig. 1 for incident energies from 0.5 to $9 \mathrm{MeV}$ and reaction angles from 0 to $180^{\circ}$. The reaction has normal kinematics for reaction angles smaller than $95^{\circ}$ at small incident energies and $120^{\circ}$ at high incident energies. Normal kinematics means the proton energy increases with increasing ${ }^{3} \mathrm{He}$ energy. The reaction has inverse kinematics for reaction angles larger than $95^{\circ}$ at small incident energies and $120^{\circ}$ at high incident energies. Inverse kinematics means that the proton energy decreases with increasing ${ }^{3} \mathrm{He}$ energy.

When the incident beam proceeds from a depth $x$ to a depth $x+d x$ in the sample, then the energy difference $d E_{p}$ of protons originating from $x$ and from 


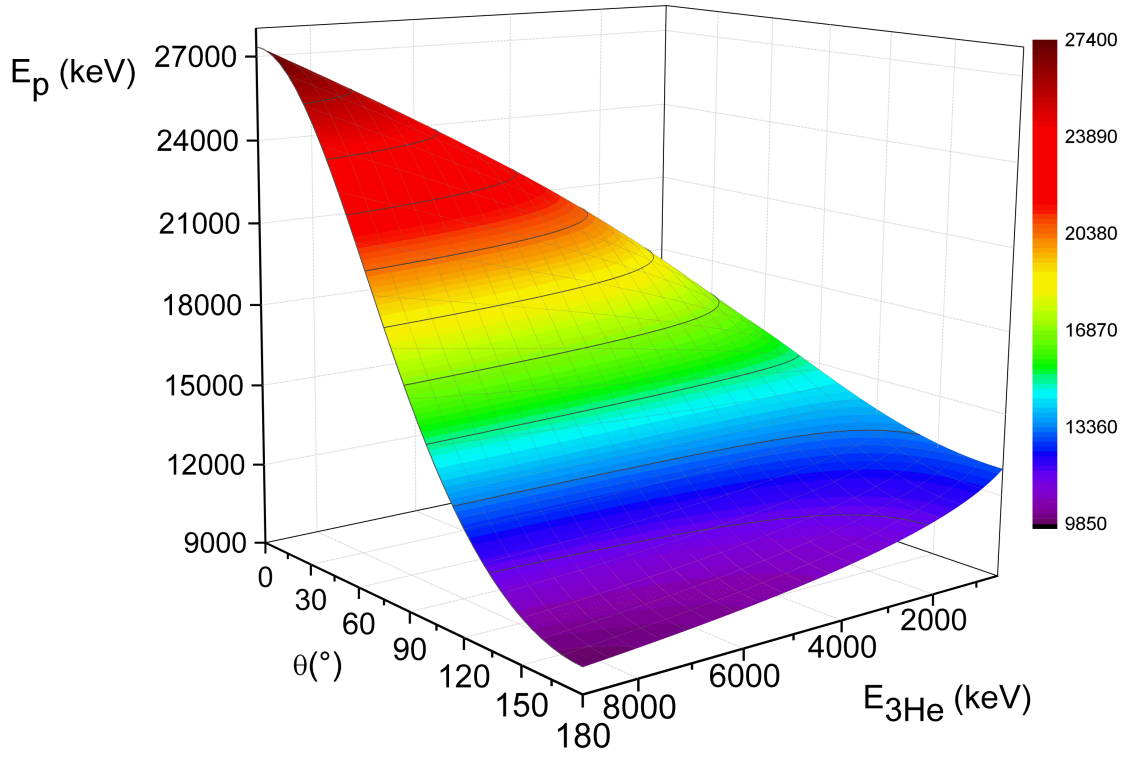

Figure 1: Energy of the protons $\mathrm{E}_{p}$ from the $\mathrm{D}\left({ }^{3} \mathrm{He}, \mathrm{p}\right) \alpha$ reaction as function of reaction angle $\theta$ and incident ${ }^{3} \mathrm{He}$ energy $\mathrm{E}_{3 \mathrm{He}}$. 
$x+d x$ is given by

$$
d E_{p}=\left[\frac{\partial E_{p}}{\partial E_{3 H e}} \frac{S_{3 H e}}{\cos \alpha}+\frac{S_{p}}{\cos \beta}\right] d x
$$

with $\alpha$ as incident angle and $\beta$ as exit angle. $\alpha, \beta$ are measured with respect to the sample surface normal. The sum of the three angles $\alpha, \beta, \theta$ gives $180^{\circ}$. $S_{3 H e}$ and $S_{p}$ are the stopping powers of the incident ${ }^{3} \mathrm{He}$ ions and of the emitted protons, respectively. $\mathrm{E}_{p}$ is the proton energy as function of ${ }^{3} \mathrm{He}$ energy $\mathrm{E}_{3 \mathrm{He}}$ and reaction angle $\theta . \partial \mathrm{E}_{p} / \partial \mathrm{E}_{3 H e}$ is the partial derivative of $\mathrm{E}_{p}$ with respect to the ${ }^{3} \mathrm{He}$ energy $E_{3 H e}$. Eq. 2 can be written shorter with the energy loss factor $[S]$, also called effective stopping power:

$$
d E_{p}=[S] d x
$$

The stopping power of the emitted high-energetic protons $S_{p}$ is generally small compared with the stopping power of the incident ${ }^{3} \mathrm{He}$ ions as obtained from previous calculations. For all materials at all practical energies the following relation applies:

$$
S_{3 H e} \geq 28 S_{p}
$$

The term $\partial E_{p} / \partial E_{3 H e}$ in eq.2 is practically always $\geq 0.5$ as later calculations will show, except in the vicinity of the transition point from inverse to normal kinematics where it approaches zero. However, as the depth resolution is worst at this point, this is irrelevant when seeking the optimal depth resolution. For $\cos \beta<0.5$, i.e. $\beta>60^{\circ}$, which is the case for all three used angles, the second term in eq. 2 can be neglected. In the following we consider normal incidence i.e. $\alpha=0^{\circ}$ (because we want to analyse large depths). The effective stopping 
power can be approximated as,

$$
[S] \approx\left[\frac{\partial E_{p}}{\partial E_{3 H e}} S_{3 H e}\right]
$$

With this we get for the proton energy change with depth $d x$ :

$$
d E_{p} \approx\left[\frac{\partial E_{p}}{\partial E_{3 H e}} S_{3 H e}\right] d x
$$

$d E_{p}$ is positive for normal and negative for inverse kinematics.

Energy loss is always associated with energy spread due to electronic energy loss straggling and multiple small-angle scattering in the sample. Moreover, the detector system adds energy spread by the finite energy resolution of the detector, by the finite size of the detector aperture which leads to geometrical straggling, and by energy-loss straggling in the stopper foil. All contributions to the energy spread originating from the sample and from the detector are summarized as $\delta E_{p}$. This converts to a minimum depth difference which can be resolved: This is called the depth resolution $\delta x$. With eq. 3 the depth resolution is given by [11]:

$$
\delta x=\frac{\delta E_{p}}{S} .
$$

As already shown in [2] the depth resolution for deuterium depth profiling using the $\mathrm{D}\left({ }^{3} \mathrm{He}, \mathrm{p}\right) \alpha$ reaction is usually limited by angular spread due to geometrical straggling or multiple small-angle scattering. Other mechanisms limiting the depth resolution, such as energy-loss straggling or the detector resolution, can therefore be neglected. For a small angular spread $\Delta \phi$ the energy spread can be approximated as

$$
\delta E_{p}=\frac{\partial E_{p}}{\partial \theta} \Delta \phi
$$


$\Delta \phi$ can represent the opening angle of the detector aperture or the width of the angular spread distribution caused by multiple scattering. With the energy spread from eq. 8 and the effective stopping power from eq. 5 the depth resolution can be calculated using eq. 7:

$$
\delta x \approx \underbrace{\frac{\left|\frac{\partial E_{p}}{\partial \theta}\right|}{\mid \frac{\partial E_{p}}{\partial E_{3 H e} \mid}}}_{\text {factor } 1} \cdot \underbrace{\frac{\Delta \phi}{S_{3 H e}}}_{\text {factor } 2} .
$$

The depth resolution given by eq. 9 consists of factor 1 which is a fraction of derivatives, multiplied by a slowly varying factor 2 .

Factor 1 is a fraction of the partial derivatives of the proton energy with respect to the reaction angle and the partial derivative of the proton energy with respect to the incident beam energy $E_{3 H e}$. With this formula the optimum angle for the detector can be derived.

The best depth resolution is achieved at energies and reaction angles where factor 1 is smallest as the other factor in eq. 9 is slowly varying. Therefore factor 1 can be seen as a proportionality factor for the depth resolution, i.e. it is proportional to the depth resolution. Figure 2 shows the numerator of factor 1. It is the absolute value of the partial derivative of the proton energy with respect to the reaction angle. This term describes the sensitivity of the proton energy at varying reaction angles. It is smallest at $180^{\circ}$ for all incident energies, meaning that at $180^{\circ}$ the proton energy hardly changes. Figure 3 shows the denominator of factor 1. This is the absolute value of the partial derivative of the proton energy with respect to the ${ }^{3} \mathrm{He}$ incident energy. It describes the change of the proton energy with respect of the chosen incident energy of the ${ }^{3} \mathrm{He}$ ions. One can clearly see a minimum in the surface in figure 3 . This minimum corresponds to the transition between normal and inverse kinematics. At the energies and angles of this valley the proton energy is the same for all incident energies, i.e., 
the protons do not carry any depth information. Putting a detector at these angles would result in no depth resolution at certain energies. The maximum of this surface is at $180^{\circ}$ for all incident energies, meaning that at $180^{\circ}$ the proton energy is most sensitive to changes of the incident energy.

Factor 1 which is proportional to the depth resolution is shown in figure 4 . The minimum of this surface is at $180^{\circ}$ which means that the best depth resolution is achieved at this angle. This angle is optimal because it has the smallest sensitivity of the emitted proton energy with respect to angular variations and at the same time offers the highest sensitivity of the proton energy with respect to changes of the ${ }^{3} \mathrm{He}$ energy, as shown above. The optimum reaction angle is optimal for all possible materials as factor 1 is material independent. Only the second factor in eq. 9 depends on sample material through the effective stopping power for the incident ${ }^{3} \mathrm{He}$ ions. This factor is only slowly varying and therefore does not affect the above considerations.

Installation of a detector at exactly $180^{\circ}$ is difficult in practice because this is the direction of the incident beam. It is only possible when using magnetic fields as done, for example, in [12]. Nevertheless, the detector should be installed as close as is technically feasible to $180^{\circ}$ due to the deterioration of the depth resolution, especially at high incident energies when moving away from this optimum angle.

The depth resolution is worst at the transition point between inverse and normal kinematics, which is at a reaction angle of about $95^{\circ}$ at low energies and at about $120^{\circ}$ at $9 \mathrm{MeV}$. Another optimum occurs at a reaction angle of $0^{\circ}$ (not shown in the figures). This minimum is only of little practical use as it implies detection of the created protons through the sample at $0^{\circ}$. While this is possible for thin foil targets it becomes impossible for thick targets with typical thicknesses in the range of $0.5-1 \mathrm{~mm}$. 


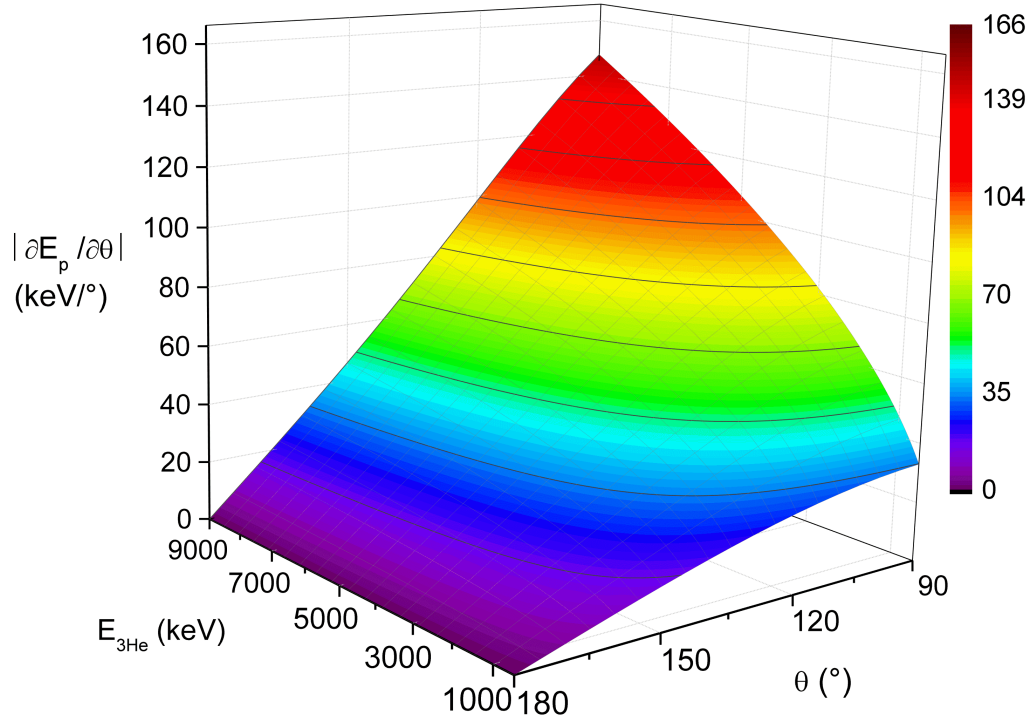

Figure 2: Absolute value of the partial derivative of the proton energy with respect to the reaction angle $\left(\mathrm{keV} /{ }^{\circ}\right)$ at diiferent incident energies $\mathrm{E}_{3 H e}$. This derivative describes the sensitivity of the proton energy with respect to the reaction angle. This derivative is the proportionality factor of the energy spread caused by angular spread, see eq. 8 .

From figure 4 one can recognize that the depth resolution gets zero at the optimized reaction angles, i.e. an infinitely good depth resolution is achieved. But it should be kept in mind that only the dominant process, i.e. angular spread, was taken into account for deriving eq. 9. Close to the optimum angles the influence of angular spread gets small, and other energy-spread processes (such as electronic energy-loss straggling in the sample or stopper foil and finite detector resolution) will become dominant. 


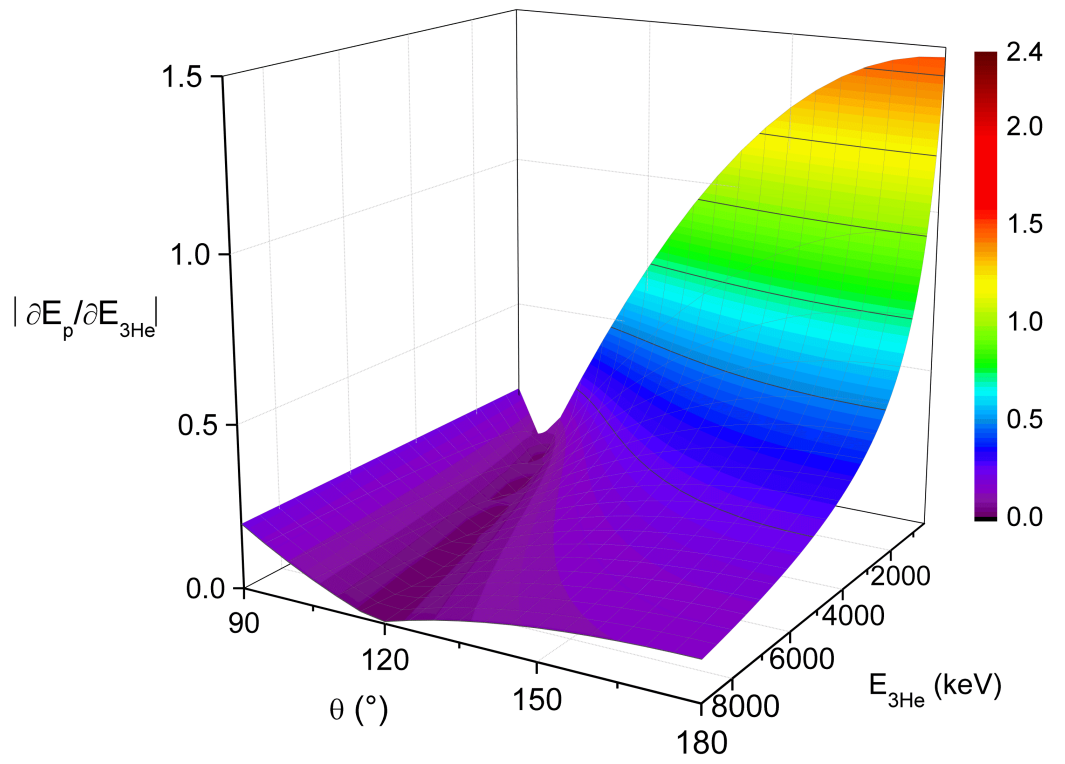

Figure 3: Absolute value of the partial derivative of the proton energy with respect to the incident ${ }^{3} \mathrm{He}$ energy at different reaction angles. This derivative describes the sensitivity of the proton energy with respect to the incident energy. The minimum in the surface is the region of the turn over from normal to inverse kinematics. 


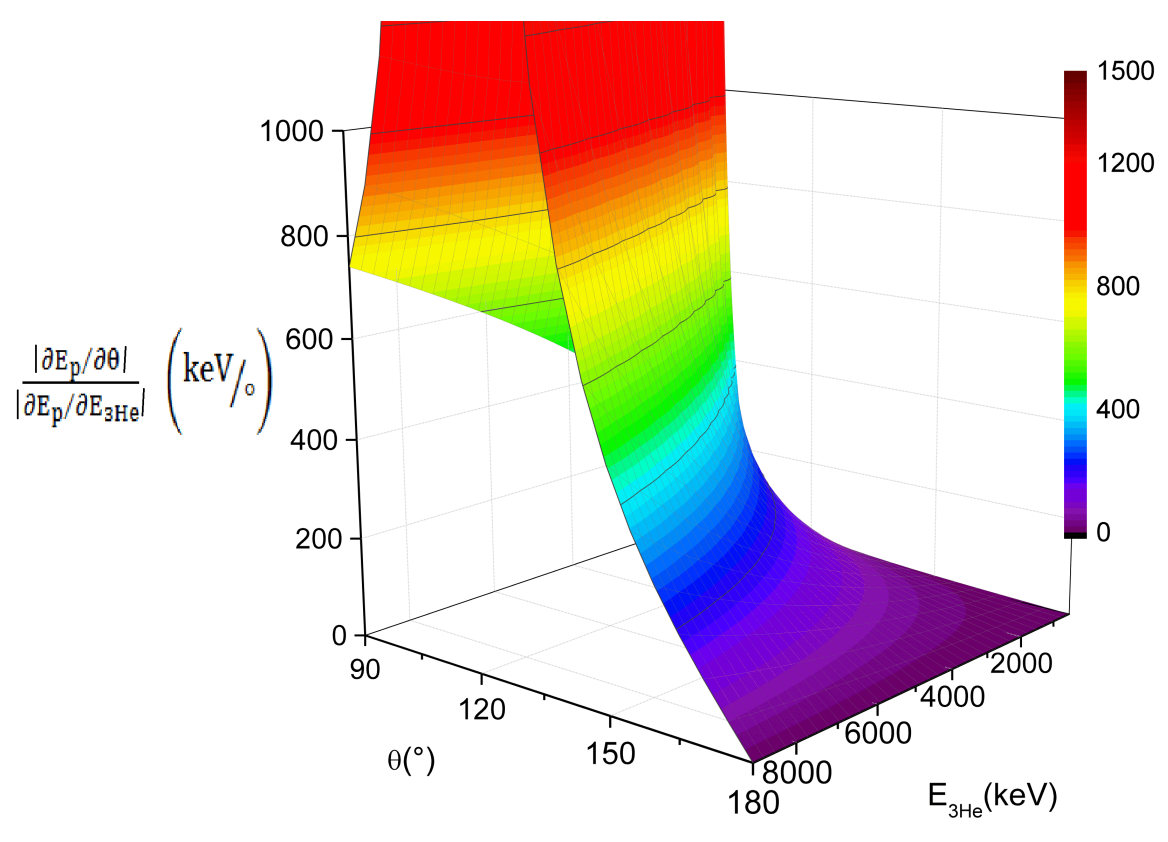

Figure 4: Absolute value of the factor 1 from eq.9. It corresponds directly to the depth resolution. The minimum of the surface is at $180^{\circ}$ for all incident energies which means that at $180^{\circ}$ the best, i.e., lowest depth resolution is achieved. 


\section{Experimental determination of the depth resolution}

\subsection{Experimental set-up}

To check this theoretical prediction and to measure the depth resolution, the energy spread for two detectors at $135^{\circ}$ and at $175.9^{\circ}$ was measured. The energy spread was then divided by the calculated effective stopping power to derive the depth resolution.

All measurements were performed in the ion beam analysis laboratory of the E2M division at the Max-Planck-Institut für Plasmaphysik. The ion beam was generated by a $3 \mathrm{MV}$ tandem accelerator. The accelerator terminal voltage was calibrated using the ${ }^{27} \mathrm{Al}(\mathrm{p}, \gamma)^{28} \mathrm{Si}$ resonances at 992 and $1380 \mathrm{keV}$ and with the ${ }^{16} \mathrm{O}(\alpha, \alpha){ }^{16} \mathrm{O}$ resonance at 3036 and $3877 \mathrm{keV}$. The nominal beam energy is correct within $0.4 \%-0.5 \%$. The beam energy spread is below $0.1 \%$ for protons at $1000 \mathrm{keV}$ and it is assumed that the spread is of the same order for higher energies.

The proton energy spectra from the nuclear reaction were recorded simultaneously by a detector at $135^{\circ}$ and a detector at $175.9^{\circ}$. At the same time backscattered ${ }^{3} \mathrm{He}$ ions were detected by a detector at $165^{\circ}$. All installed detectors are semi-conductor detectors with different depletion depths according to their intended application.

The proton detectors are partially depleted silicon surface barrier detectors. The proton detector located at $135^{\circ}$ has a parabolic slit with a slit width of $3 \mathrm{~mm}$ and a height of $17 \mathrm{~mm}[2]$ resulting in $135^{\circ} \pm 2.3^{\circ}$. The detector to target distance is $37.3 \mathrm{~mm}$. The measured solid angle is $30.26 \mathrm{msr}$. The detector depletion depth is $2000 \mu \mathrm{m}$. The foil in front of the detector consists of a $12 \mu \mathrm{m}$ thick Mylar foil coated with $10 \mathrm{~nm}$ gold and a $5 \mu \mathrm{m}$ thick nickel foil.

The proton detector at $175.9^{\circ}$ is an annular detector with a depletion depth of $2000 \mu \mathrm{m}$. Annular means it has a hole in the center for the incident beam. The 
detector to target distance is $84 \mathrm{~mm}$. The aperture in front of the detector has an inner diameter of $7 \mathrm{~mm}$ and an outer diameter of $16 \mathrm{~mm}$. This results in an angle range from $174.6^{\circ}$ to $177.6^{\circ} .175 .9^{\circ}$ is the mean angle of the detector weighed with the detector's active surface. Its experimental solid angle is $17.5 \mathrm{msr}$. In front of the annular detector a $50 \mu \mathrm{m}$ thick Mylar foil coated with a $50 \mathrm{~nm} \mathrm{Au}$ film is positioned.

The detector at $165^{\circ}$ for the ${ }^{3} \mathrm{He}$ backscattered ions is a passivated implanted planar silicon model and has a depletion depth of $504 \mu \mathrm{m}$ and is located at a distance of $64.1 \mathrm{~mm}$ from the target. The solid angle resulting from calibration measurements based on four different IRMM targets is $1.101 \pm 0.038 \mathrm{msr}$.

For a precise determination of the collected charge the target is surrounded by a negatively-biased Faraday shield and a grounded shield.

\subsection{Samples}

To measure the energy spread in different depths and materials, samples were prepared with a thin deuterium containing carbon layer buried under different materials with different thicknesses. For measuring the energy spread, a sample is needed with a deuterium containing layer which has a sufficiently small thickness so that the energy spread due to the layer thickness is small compared to the energy spread caused by all other processes. In such a case the width of the proton peak is the sought energy spread.

As deuterium-containing target a dense, thin plasma-deposited amorphous deuterated carbon (a-C:D) film was grown on the driven electrode of an asymetric capacitively coupled RF discharge. As substrates three crystalline silicon (100) wafers were used labelled as wafer 1, 2, 3 in the following. For deposition

a $\mathrm{CD}_{4}$ flow of $30 \mathrm{sccm}$ and a RF sputter power of $43 \mathrm{~W}$ leading to a DC self bias of $300 \mathrm{~V}$ was used. The pressure during deposition was $2 \mathrm{~Pa}$. The deposition rate was about $5 \mathrm{~nm} / \mathrm{min}$. The $\mathrm{Si}$ wafer was sputter-cleaned with $\mathrm{Ar}$ ions prior 


\begin{tabular}{|c|c|c|}
\hline Si wafer & $\begin{array}{c}\text { Thickness of the a-C:D layer } \\
(\mathrm{nm})\end{array}$ & $\begin{array}{c}\text { D areal density } \\
\left(10^{15} \frac{\mathrm{at}}{\mathrm{cm}}\right)\end{array}$ \\
\hline 1 & $79 \pm 4$ & 440 \\
\hline 2 & $79 \pm 4$ & 401 \\
\hline 3 & $79 \pm 4$ & 448 \\
\hline
\end{tabular}

Table 1: Thicknesses of the a-C:D layers deposited on three different Si wafer. The thicknesses (in $\mathrm{nm}$ ) were measured by profilometry at several points of the wafer. The D areal densities were determined by NRA at several energies and at several points on the wafer as well.

to the deposition in order to achieve a better adhesion between the a-C:D layer and the Si wafer. The $\mathrm{D} /(\mathrm{C}+\mathrm{D})$ ratio was assumed to be about 0.34 which is consistent with a-C:H films deposited under the same conditions [13].

In order to measure the a-C:D layer thickness by profilometry a line was painted on the wafer with a permanent marker. After deposition the permanent marker was removed with alcohol leaving a sharp edge between the coated and uncoated areas. The thickness of the a-C:D layer was measured with a tactile profilometer at several points on the wafer. It was $79 \mathrm{~nm} \pm 4 \mathrm{~nm}$. The D areal densities of the a-C:D layers were determined by means of NRA using the $\mathrm{D}\left({ }^{3} \mathrm{He}, \mathrm{p}\right) \alpha$ reaction at different energies and points on the sample. The spectra were simulated using the SIMNRA program [14]. The thicknesses and D areal densities of the a-C:D layers are listed in table 1. All three a-C:D layers have identical interference colors which means that they are homogeneous and the thickness variation between them is below $10 \%$ as the measured data in table 1 verify.

In order to measure the depth resolution in different materials the a-C:D/Si wafers were cut into smaller pieces and covered with different cover metal layers. Aluminum was chosen as a low $\mathrm{Z}$ material. As a medium $\mathrm{Z}$ material molybdenum was used. Tungsten was chosen as a high $\mathrm{Z}$ material. To determine the depth resolution in different depths, samples with different metal cover layer thicknesses were deposited. Aluminium was deposited on the pieces of wafer 
1. Molybdenum was deposited on wafer 2 and tungsten on wafer 3 . The layers were deposited on top of the a-C:D films in a sputter device. The nominal material thicknesses of the metal cover layers were $0.5 \mu \mathrm{m}, 2 \mu \mathrm{m}, 5 \mu \mathrm{m}, 8 \mu \mathrm{m}$. For Aluminium additionally a $11 \mu \mathrm{m}$ thick layer was deposited.

All metal cover layers were deposited by DC magnetron sputtering in the Denton device at IPP. For the deposition of $\mathrm{Al}$ a $\mathrm{DC}$ power of $500 \mathrm{~W}$ was set on the $45.6 \mathrm{~cm}^{2}$ cathode which resulted in a target voltage of $450 \mathrm{~V}$. The Ar flow was set to be $60 \mathrm{sccm}$ and the pressure during deposition was $0.9 \mathrm{~Pa}$. The $\mathrm{Al}$ deposition rate was $53.0 \mathrm{~nm} / \mathrm{min}$. For the deposition of Mo a DC mode of $200 \mathrm{~W}$ was used, the target voltage was $292 \mathrm{~V}$. The Ar flow was $80 \mathrm{sccm}$ and the pressure during deposition was 1.5 $\mathrm{Pa}$. The deposition rate of Mo was $25.0 \mathrm{~nm} / \mathrm{min}$. For the deposition of $\mathrm{W}$ a DC mode of $200 \mathrm{~W}$ also was used. Here the target voltage was $360 \mathrm{~V}$. The Ar flow was $80 \mathrm{sccm}$ and the pressure during deposition was $1.5 \mathrm{~Pa}$. The deposition rate was $15.3 \mathrm{~nm} / \mathrm{min}$. In all three depositions the substrate was on floating potential. In order to achieve a better adhesion between the a-C:D layer and the Mo and W-layers the aC:D was sputtered with Ar for 1 min before the actual deposition started. This process removes impurities from the film surface and improves the adhesion. The amount of the sputtered a-C:D can be neglected.

To be able to measure the thickness of the metal cover layers with a tactile profilometer a part of the samples was covered with tape to produce a sharp edge between the a-C:D and the sputtered layer. The measured thicknesses by the profilometer are tabulated in table 2 . The error is in the worst case about $\pm 50 \mathrm{~nm}$. This error contains the uncertainty of the measurement by profilometry and also the uncertainty resulting from the fact that the edge was not perfectly sharp in some cases.

The areal density was determined by means of RBS with ${ }^{3} \mathrm{He}$ as incident 
ions at several energies and in an additional RBS measurement with protons as incident ions with an energy of 3000 and $4000 \mathrm{keV}$. The mean values of the measurements are given in table 2. The error is estimated to be $\pm 300 \cdot 10^{15} \frac{\mathrm{at}}{\mathrm{cm}^{2}}$ in the worst case. This error was estimated based on the scatter of the individual measurements. With the thickness measured by profilometry and the areal density measured by RBS, the atomic density in at $/ \mathrm{cm}^{3}$ of the layers can be deduced. The experimental density deviates from the theoretical density within less than $12 \%$, see table 3 . As shown in[15] layer deposition by sputtering can produce layers with slightly reduced density. Therefore, the densities of all three metal layers were determined experimentally. Moreover, small amounts of the sputter gas Ar and oxygen can be incorporated in the layers, thus further reducing the atomic density. Ar can be here neglected as no Ar was observed in the RBS spectra.

The oxygen concentration in the layers was determined from the RBS spectra. While low concentrations of oxygen are not directly visible in the spectra, the height of the RBS metal layer peak will decrease if oxygen is contained in the metal layer. From this reduction in height the oxygen concentration can be calculated. Only for the Mo samples the oxygen peak was visible directly in the RBS measurements with protons. For these samples the oxygen ratio was also determined directly from the oxygen peak.

The mean values of the determined oxygen concentrations are shown in table 2. For the three $\mathrm{Al}$ layers with $4,7,11 \mu \mathrm{m}$ the oxygen concentrations could not be deduced from the RBS spectra because the signals from Si and Al overlap. The absolute error in the oxygen concentration determination is \pm 2.5 at. $\%$ (i.e. for the determined oxygen concentration of $12 \%$ the uncertainty range is between $9.5 \%$ and $14.5 \%)$ and in the worst case \pm 5 at.\%. 


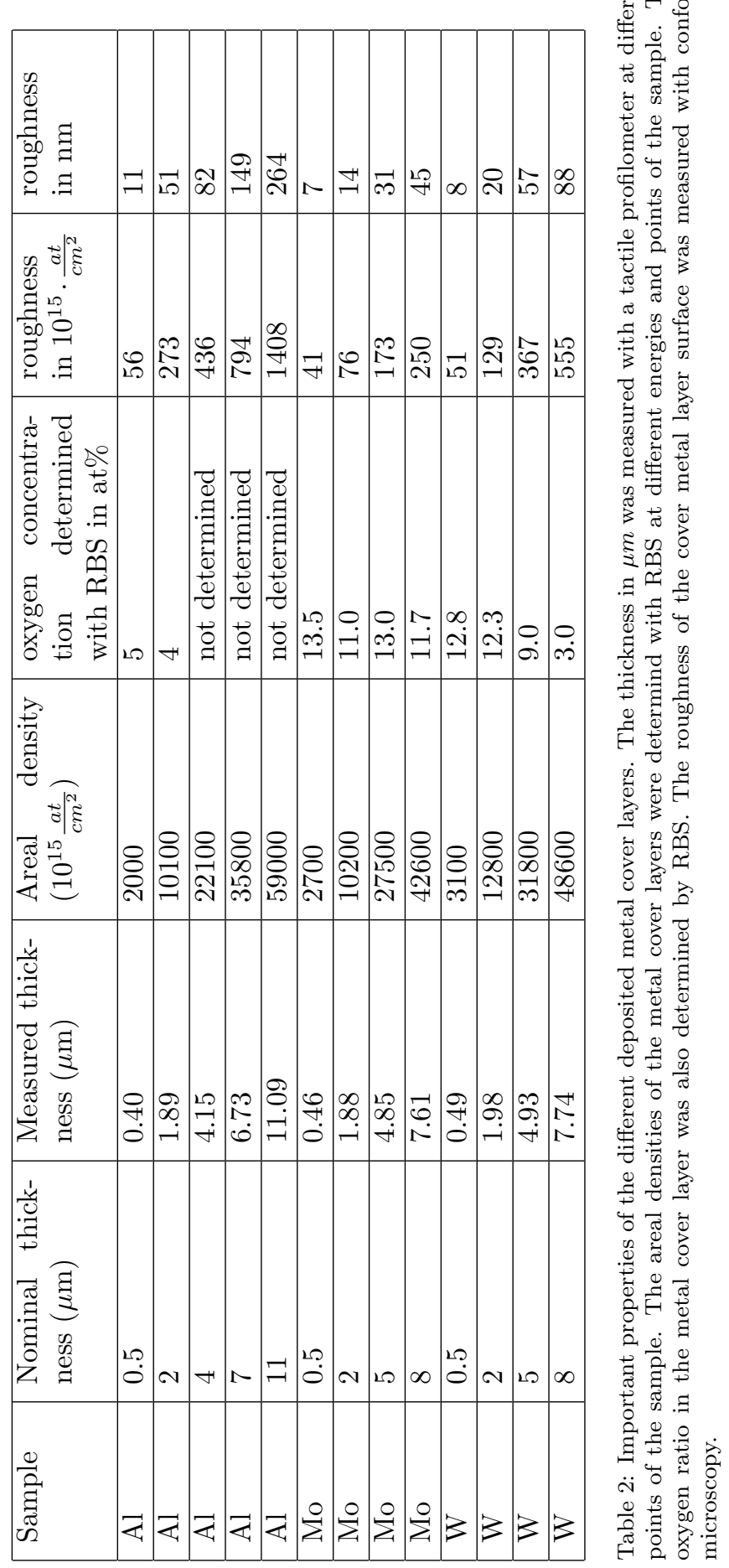




\begin{tabular}{|l|l|l|l|}
\hline Material & $\begin{array}{l}\text { Experimental } \\
\left(10^{22} \frac{a t}{c^{3}}\right)\end{array}$ & $\begin{array}{l}\text { Theoretical } \\
\left(10^{22} \frac{a t}{c m^{3}}\right)\end{array}$ & Ratio \\
\hline $\mathrm{Al}$ & 5.324 & 6.024 & 0.88 \\
\hline $\mathrm{Mo}$ & 5.645 & 6.409 & 0.88 \\
\hline $\mathrm{W}$ & 6.413 & 6.319 & 1.02 \\
\hline
\end{tabular}

Table 3: Experimental and theoretical atomic densities of the deposited metal layers. The latter were calculated from the determined areal density of the metal layer from RBS and the thickness from profilometry. The third row shows the theoretical densities. The fourth row contains the ratio between the experimental and theoretical values.

\subsection{Roughness of the samples}

For sufficiently thin a-C:D layers the width of the proton peak in the NRA spectra is the sought energy spread induced by the cover metal layer. This is, however, only true for perfectly smooth surfaces of the cover metal layers. If the cover layers' surfaces are rough, i.e. if the layer thickness varies, then the incident and outgoing particles pass through different thicknesses: This causes an additional energy spread of the proton peak. It is therefore important to know whether the roughness of the cover metal layer influences the peak width significantly, i.e. the layers must be sufficiently smooth so that the influence of layer roughness is considerably smaller than the influence of all other energy spread mechanisms. The roughness of the metal layers was measured by confocal microscopy. An image was taken of each sample surface. In the next step surface profiles were generated by the microscope software at ten different lines on the sample, see figure 5 .

The roughness is defined as the full width at half maximum (FWHM) of the deviation of the real surface profile from an ideal smooth surface. Because the individual samples have a slight curvature, a parabola was fitted to the measured surface profile at ten different positions on each sample to represent an ideal smooth surface, see figure 5. The deviation between the measured profile and this fit curve was calculated for points at intervals of $125 \mathrm{~nm}$. These calculated deviations from the ten curves were plotted as a sum histogram for each 


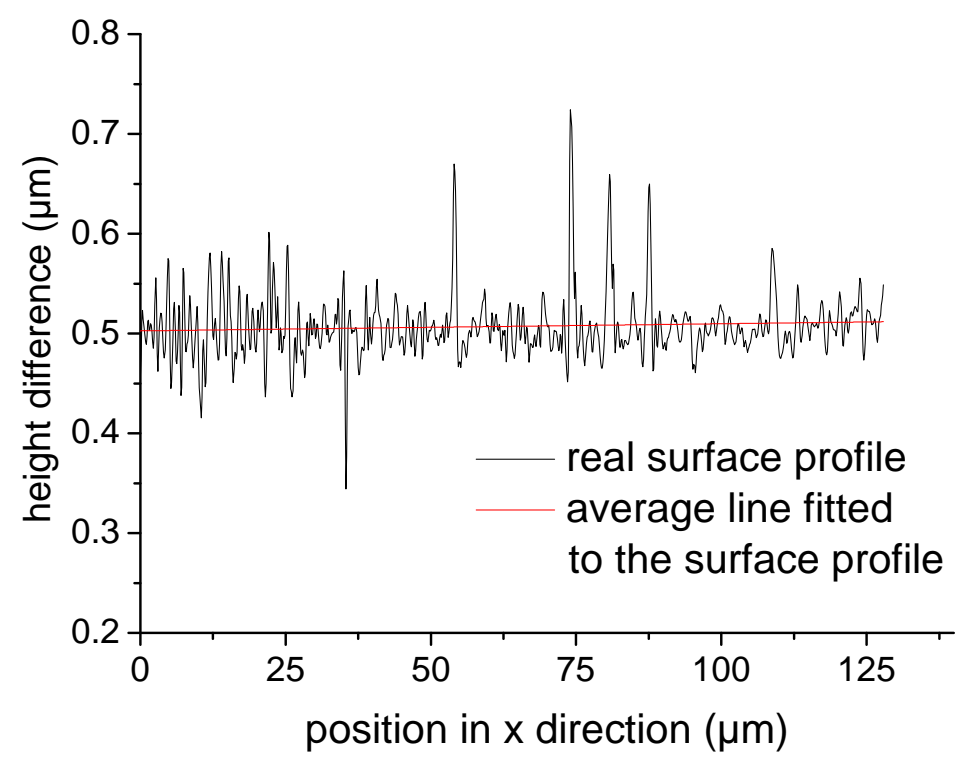

Figure 5: The real surface profile of the sample with $\approx 2 \mu \mathrm{m} \mathrm{Al}$ on top of a-C:D on Si wafer is shown. An line was fitted to the surface profile to represent a smooth surface.

sample, see figure 6. The shape of the histograms reminds a shifted Gaussian distribution. Due to this reason a Gaussian with position and width as free parameter was fitted to the histograms to determine their FWHM, see figure 6 .

The such determined roughnesses of the different samples are tabulated in table 2 . The roughness is typically $2 \%-3 \%$ of the layer thickness.

To check whether this roughness changes the width of the proton peak a simulation with SIMNRA was performed for each sample. The NRA spectra were simulated with the layer areal densities as given in table 2. One simulation set was performed assuming a smooth surface and the other simulation set was done with the rough surfaces as listed in table 2. The proton peak resulting in a simulation with a smooth surface and the proton peak resulting from a rough surface are shown in figure 7 for the sample with a $2 \mu \mathrm{m} \mathrm{Al}$ cover layer. The proton peaks have nearly identical widths. For all samples the widths 


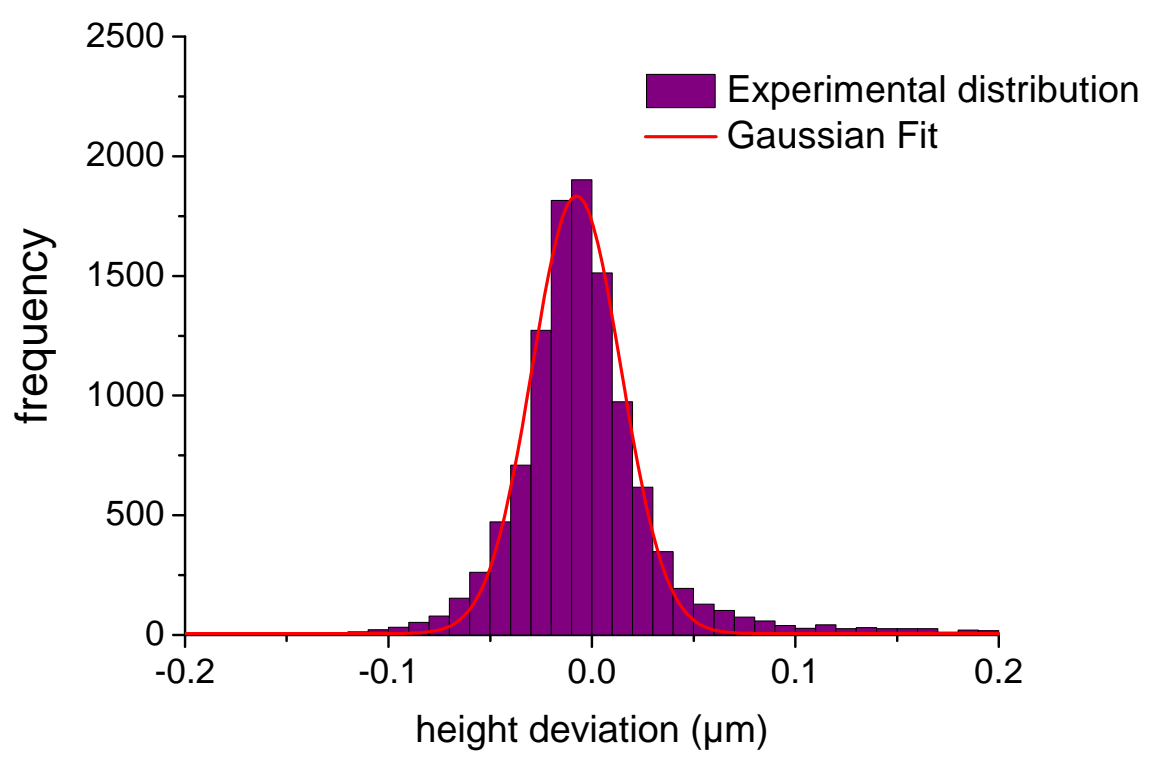

Figure 6: Histogram of the deviations from a smooth surface with a gaussian fit is shown for the sample with $\approx 2 \mu \mathrm{m} \mathrm{Al} / \mathrm{a}-\mathrm{C}: \mathrm{D} / \mathrm{Si}$. With the width of the histogram the roughness of the metal cover layer surface was estimated. 
of the proton peaks were identical within a few percent. The additional energy spread due to the roughness (as determined by confocal microscopy) is therefore insignificant.

The roughnesses were also determined from the RBS spectra by simulating the widths of the metal peaks. For rough layers the low energy edge gets broader. The roughnesses of the metal cover layers as determined by RBS were larger than the measured ones by profilometry. For the $\mathrm{Al}$ and $\mathrm{W}$ layers the roughnesses were larger by a factor of typically $3-4$ than in table 2 . To be sure that even this larger roughnesses of the samples does not influence the widths of the peaks, simulations were carried out with these roughnesses and the FWHMs of proton peaks were compared to the simulations without roughness. The FWHM changes only insignificantly even with these larger roughnesses, as shown in figure 7 for one example. Therefore it can be concluded that the influence of the roughness of the metal cover layers is negligible for all performed depth resolution measurements.

The structure of the samples surfaces was investigated by scanning electron microscopy (SEM) using a Helios device from FEI company. Typical top views of the sample surfaces are shown in figure 8. For imaging secondary electrons were used. At some places on the metal cover layers larger crystallites are observed, see $8 \mathrm{c}$ ), e). These crystallites have a diameter of about $3-5 \mu \mathrm{m}$ and cover about $2 \%$ of the surface area. These larger crystallites can result in a high energy tail in the proton energy spectra but do not change the width of the proton peak significantly as they are relatively few. As can be also seen in the SEM images the lateral scale length of the roughness is below $1 \mu \mathrm{m}$. This variation is smaller than the laser beam spot size of the confocal microscope. The confocal microscope can not resolve the roughness variations below $1 \mu \mathrm{m}$ and this might be the reason why different roughnesses are observed by confocal 


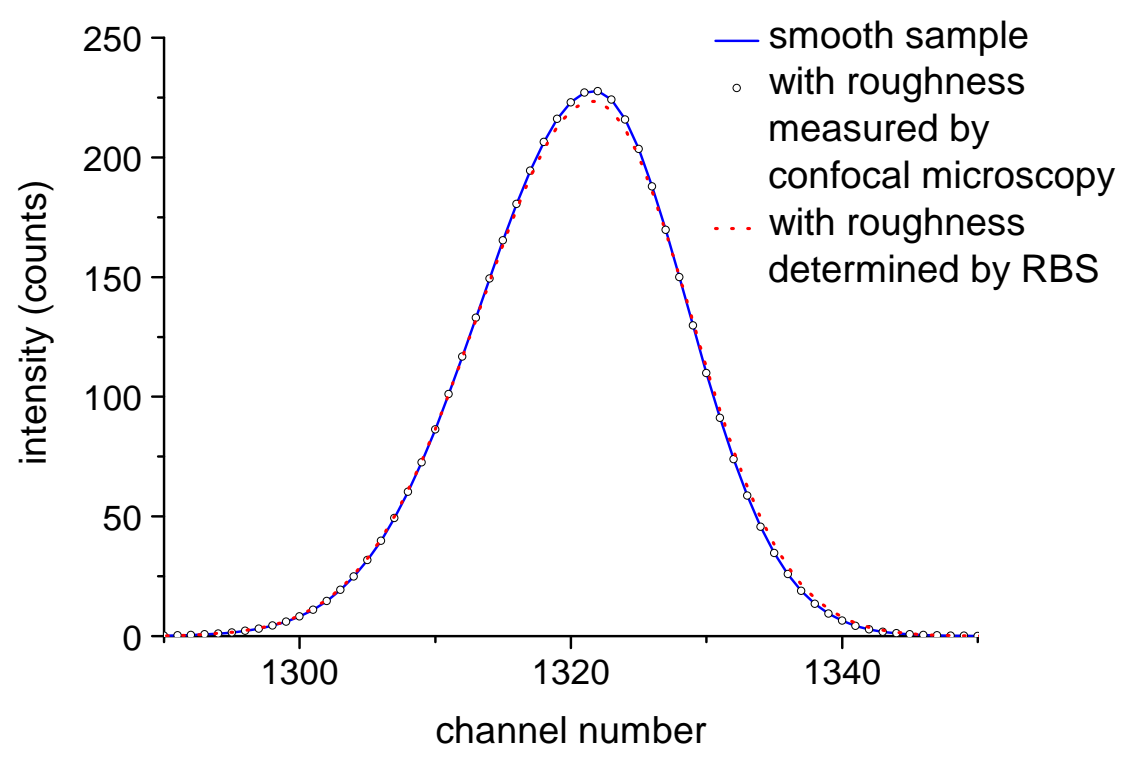

Figure 7: Simulated proton peak of the sample with $\approx 2 \mu \mathrm{m} \mathrm{Al} / \mathrm{a}-\mathrm{C}: \mathrm{D} / \mathrm{Si}$. The blue line is the simulated proton peak of an ideally smooth $\mathrm{Al}$ surface. The dots represent the simulated proton peak of a rough $\mathrm{Al}$ surface. For this simulation we used the roughness measured with the confocal microscope. The dashed red line is the simulated proton peak of a more rough $\mathrm{Al}$ surface using the roughness determined by RBS. 
microscopy and by the RBS measurements.

\subsection{Results}

To determine the energy spread caused by the metal cover layers on top of the a-C:D layers, energy spectra of protons from the $\mathrm{D}\left({ }^{3} \mathrm{He}, \mathrm{p}\right) \alpha$ nuclear reaction were recorded for each sample at different incident energies ranging from $0.5 \mathrm{MeV}$ to $6 \mathrm{MeV}$ with a step width of $0.5 \mathrm{MeV}$ at reaction angles of $135^{\circ}$ and $175.9^{\circ}$. The energy spectra of both detectors were measured simultaneously in the same setup. A collected charge of $5 \mu \mathrm{C}$ was used for each measurement. In order to minimize the influence of deuterium depletion by ion-bombardment induced desorption, different spots on the target were used at each energy.

Figure 9 shows typical spectra from the sample with about $0.5 \mu \mathrm{m} \mathrm{Al}$ on a-C:D on Si for incident energies of 1, 2.5 and 4.5 MeV. The protons from the $\mathrm{D}\left({ }^{3} \mathrm{He}, \mathrm{p}\right) \alpha$ reaction are visible at $12-13 \mathrm{MeV}$ energy. At $1 \mathrm{MeV}$ incident energy only protons from this reaction are visible. At $2.5 \mathrm{MeV}$ incident energy additional peaks from the ${ }^{12} \mathrm{C}\left({ }^{3} \mathrm{He}, \mathrm{p}_{x}\right){ }^{14} \mathrm{~N}$ reaction start to become visible between 1.5 and $5.5 \mathrm{MeV}$ and at $4.5 \mathrm{MeV}$ incident energy additional peaks from nuclear reactions with $\mathrm{Si}$ are visible. The width of the proton peak from the $\mathrm{D}\left({ }^{3} \mathrm{He}, \mathrm{p}\right) \alpha$ reaction was determined by fitting a Gaussian to the proton peak of each measurement, shown in figure 9 right hand side. With this Gaussian FWHM which is equal to the width of the proton peak the energy spread for the detectors was determined experimentally.

To compare the measured energy spread to the theoretical predictions the NRA measurements were simulated with SIMNRA using the applied experimental conditions (detector aperture width, foil thicknesses, energy calibration, etc.). The simulation was calculated for the same target composition as given in tables 1, 2. For the simulation of the proton signal from the $\mathrm{D}\left({ }^{3} \mathrm{He}, \mathrm{p}\right) \alpha$ reaction the cross sections from [4] were used. For backscattering from the cover metal 

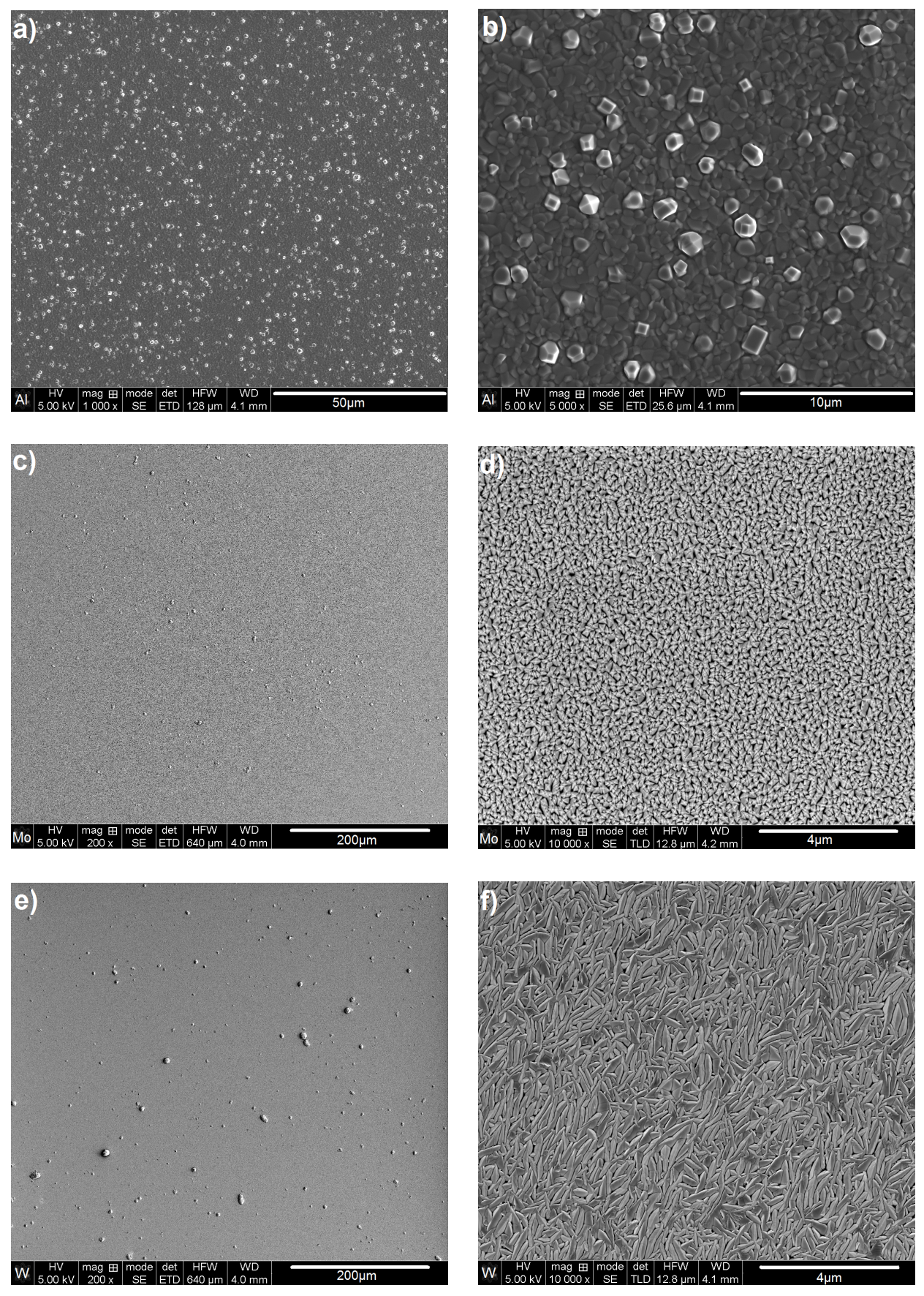

Figure 8: SEM images: Top view of the surfaces of the metal cover layers. Figures a) and b) show the surface of the $2 \mu \mathrm{m}$ thick Aluminium. Figures c) and d) show the molybdenum layer of $5 \mu \mathrm{m}$ thickness. Figures e) and f) show the surface of the $5 \mu \mathrm{m}$ thick tungsten. For all these images the secondary electron signal was used. 
layer the Rutherford cross section was used. The FWHMs of the simulated proton peaks were determined by fitting a Gaussian. With this the theoretical energy spread is known.

To calculate the depth resolution from the energy spread the effective stopping power $\mathrm{S}$ has to be known, as the depth resolution is the energy spread divided by the effective stopping power, see eq. 7 .

The effective stopping power for these samples for each incident energy was calculated by ViewNRA which is part of the SIMNRA package. The measured and simulated energy spread was then divided by the corresponding stopping power thus giving the depth resolution.

The measured depth resolutions (dots) and the simulated depth resolutions (lines) are shown in figures 10-12 for both detectors.

It can be seen that at all energies a better depth resolution is achieved with the annular detector at $175.9^{\circ}$ compared with the depth resolution achieved with the detector at $135^{\circ}$. This holds for aluminum as well as for molybdenum and tungsten. At low incident energies the depth resolutions of both detectors are very similar. At higher energies the depth resolution gets worse for both detectors. This deterioration of the depth resolution is strong for the $135^{\circ}$ detector while it is much less pronounced for the annular detector at $175.9^{\circ}$. The depth resolution achieved with the annular detector is considerably better compared to the depth resolution achieved with the $135^{\circ}$ detector, see figures 10-12. The depth resolution of the a-C:D / Si samples (figures 10a), 11a), 12a)) are calculated with the effective stopping power of an infinitesimally thin metal cover layer. This is the reason why the determined depth resolution is different between the three a-C:D / Si samples. This is the depth resolution of an infinitesimally thin metal cover layer of $\mathrm{Al}, \mathrm{Mo}$ or $\mathrm{W}$ on the a-C:D / $\mathrm{Si}$ (figures 10a), 11a), 12a)). 

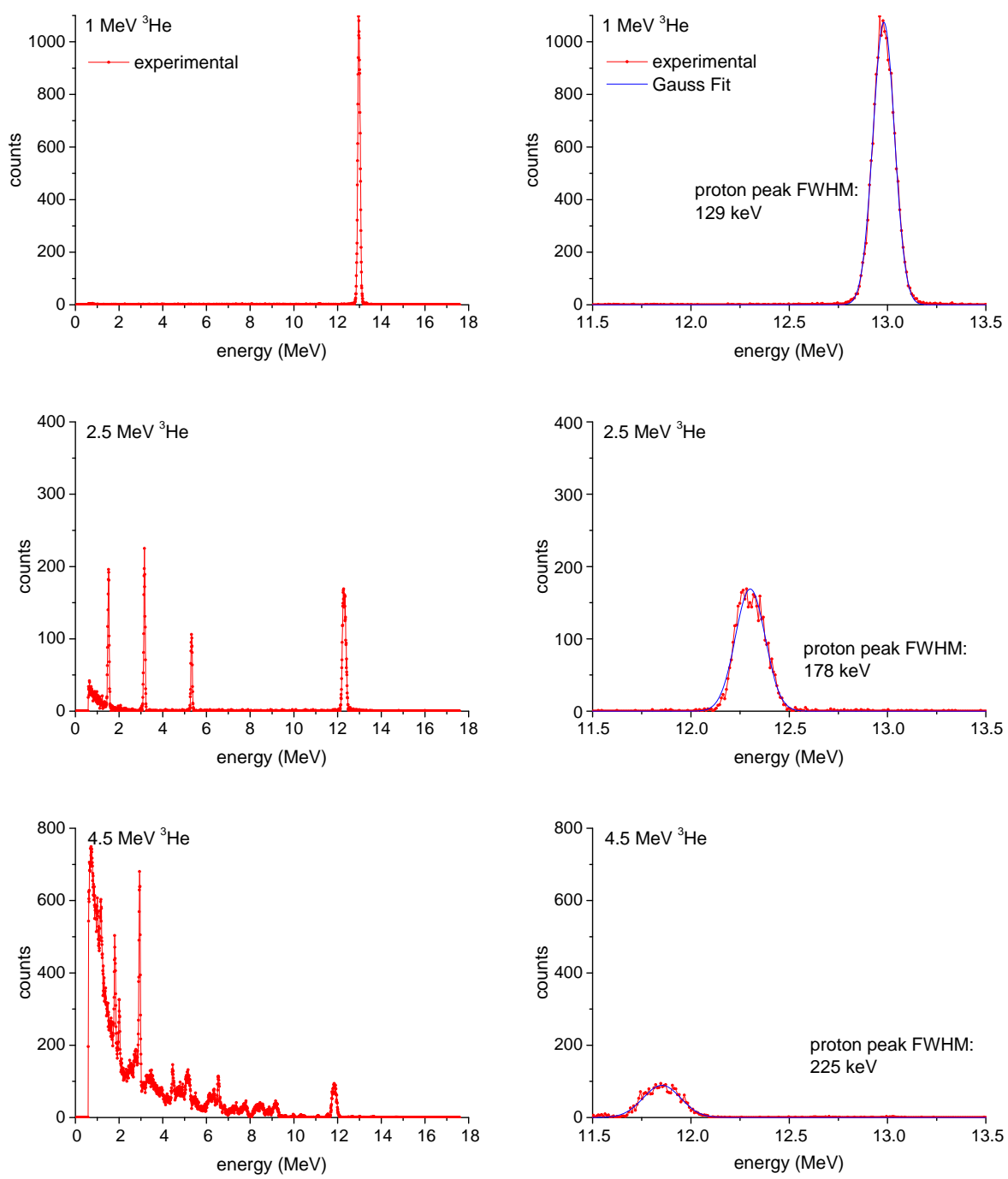

Figure 9: Proton energy spectra for the sample with a approximately $0.5 \mu m$ thick aluminium cover layer on a-C:D / Si. The lefthand side shows the full spectra. On the righthand side the region of the proton peak of the same spectra is shown. A Gaussian is fitted to the proton peak in order to determine the width of the peak. 
For thin aluminium or molybdenum metal cover layers the depth resolution gets up to $70 \mu \mathrm{m}-80 \mu \mathrm{m}$ for high incident energies at the $135^{\circ}$ detector, see figures 10a)-c),11a), b). For thin tungsten cover layers even worse depth resolution for the $135^{\circ}$ detector are observed compare figures $\left.\left.12 \mathrm{a}\right), \mathrm{b}\right)$. Please note the axis breaks in the mentioned figures. That means at these high incident energies the depth resolution is infinite, i.e. the spectra provide no depth information. The increase of the depth resolution with increasing energy is also observed at the annular detector at $175.9^{\circ}$. This increase is not that steep, i.e. the depth resolution increase up to $8 \mu \mathrm{m}$ in the worst case, figure 10e). With the annular detector we achieve for all energies a finite depth resolution.

The improvement of the depth resolution is highest for the high- $\mathrm{Z}$ material tungsten, i.e. a better depth resolution up to 18 times is reached with the annular detector in comparison to the $135^{\circ}$ detector, figure $12 \mathrm{~b}$ ). The smallest improvement of the depth resolution is observed in the low-Z material aluminium. Nevertheless the improvement is also high and up to an factor of 10, figure 10f). For the given comparison the experimental data points at the highest energies were used. This significant improvement confirms the theoretical predictions presented in section 2. The tendency of the experimental data points and of the simulations is very similar meaning that the simulations are describing the depth resolution well. However, the simulated depth resolution is somewhat lower in the whole energy range than the measured one, i.e. the simulations are slightly too optimistic. The differences between the simulations and the experimental data are lowest for the a-C:D/Si samples, figures 10a), 11a), 12a). The lower energy spread of the simulated data may be explained with the fact that the simulation does not take into account the potential roughness of the stopper foil in front of the detector. This roughness is unknown and difficult to measure for the used Mylar foils due to their sensitivity to ion irradiation. 
At the other samples the differences are increasing with increasing thickness of the cover metal layer. The increase of the difference between the simulations and the data points with thickness can't be explained with the roughness of the foil as the same foil was used during the whole measurement. This implies that the depth dependence of the processes limiting the depth resolution is not fully correct in the simulations.

Based on the measurements performed, it is impossible to state which processes limiting the depth resolution are underestimated in the simulation and which of them has an incorrect depth or energy dependence. In figures10 a)-c) the experimental data of the annular detector for the highest energies were not evaluated because of the high background. In figures 11d),e), 12b),c),e) there are experimental data points at the lowest energies but the simulations did not calculate spectra for these energies. The reason for this effect is a slightly inaccurate stopping power in the simulations.

The uncertainty of the simulations due to the uncertainties of the layer thicknesses and the oxygen amounts can be estimated. This was done exemplarily for four simulated data points. The areal density of the a-C:D layer was determined with an accuracy of $10 \%$. The areal density of the metal layers has an error margin of $\pm 300 \mathrm{at} / \mathrm{cm}^{2}$. The oxygen amount is known within $\pm 5 \%$ in the worst case. The uncertainties of the Gaussian fits and the detector resolution have an uncertainty of $4.5 \%$ for each point. To estimate the resulting uncertainties, the spectra were simulated with the layer areal densities from table 2 adding the absolute uncertainty $\pm 300 \mathrm{at} / \mathrm{cm}^{2}$ and the a-C:D layer areal density from table 1 adding $\pm 10 \%$ and the oxygen amount from table 2 adding $\pm 5 \%$. Then again a Gaussian was fitted to the simulated spectra and the FWHMs were compared. The total uncertainty is the quadratic sum of all uncertainties as they are statistically independent. 
The total uncertainty for the four points resulted to be below $7 \%$. The uncertainty of the simulation (based on the uncertainty of more peaks) for all other points should be of the same order. This uncertainty of the simulation does not explain the difference between the simulation and the experimental data. The uncertainty of the simulations caused by uncertainties in the stopping power and other processes is not possible to calculate. However the simulations describe the experimental data well. 
a)

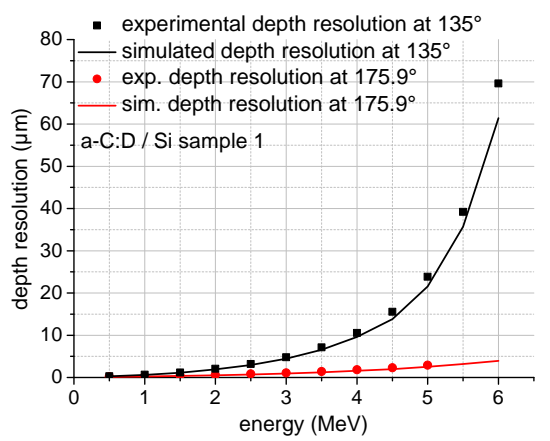

c)

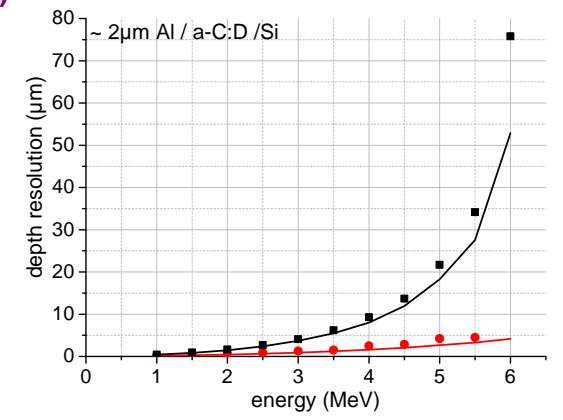

e)

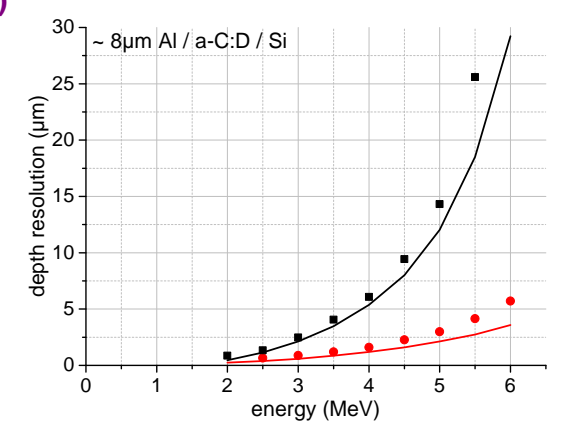

b)

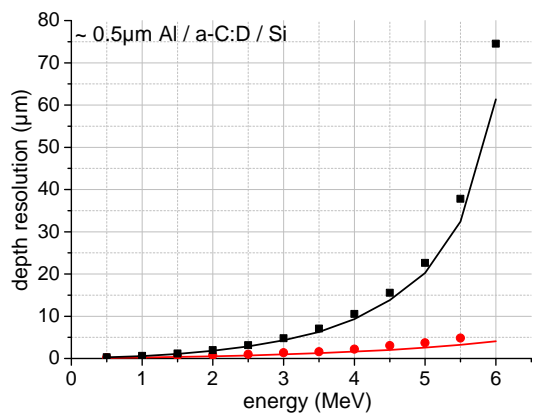

d)

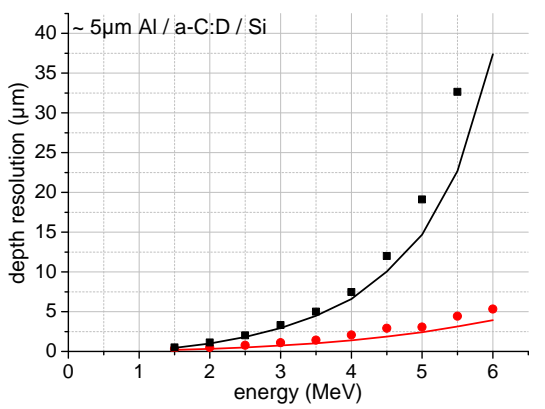

f)

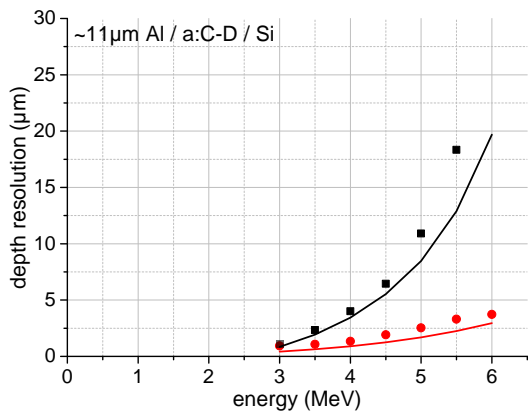

Figure 10: Depth resolution in $\mu \mathrm{m}$ versus incident ${ }^{3} \mathrm{He}$ energy in aluminium for different thicknesses of the aluminium cover layer. The dots are measured data. The lines are simulated data. The data from the annular detector at $175.9^{\circ}$ are in red. The data from the detector at $135^{\circ}$ are in black color in the figures. The depth resolution at the a-C:D / Si sample (figure 10a)) is calculated with the effective stopping power of an infinitesimally thin aluminium cover layer. Please note the different axis scales in the different figures and the axis breaks in some figures. In all above figures one can see that the better, i.e., lower, depth resolution is achieved with the detector at $175.9^{\circ}$. For thick Aluminium layers (figure 10d-f) the experimental determination of the depth resolution at $6 \mathrm{MeV}$ incident energy for the 135 detector was impossible due to a high background. Comparing the depth resolution at high energies between experimental points an improvement up to a factor of 10 is reached. 
a)

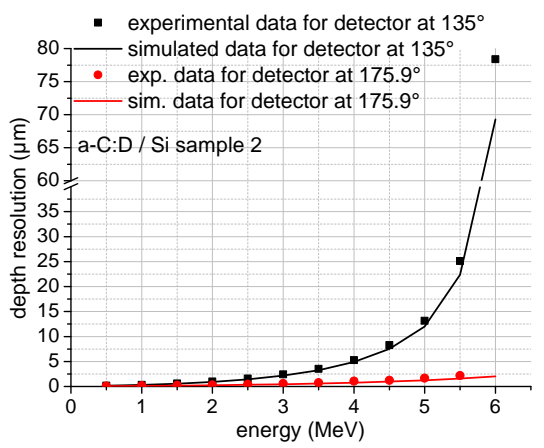

c)

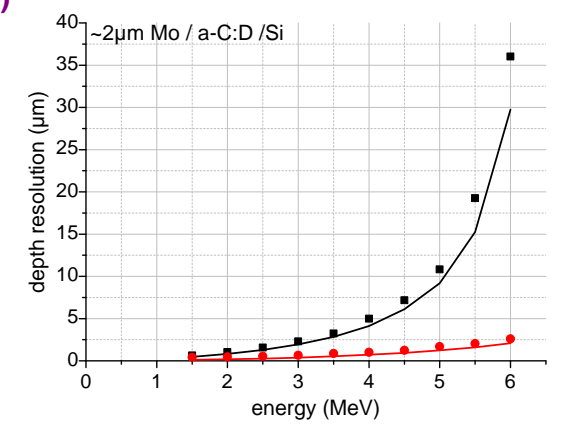

e)

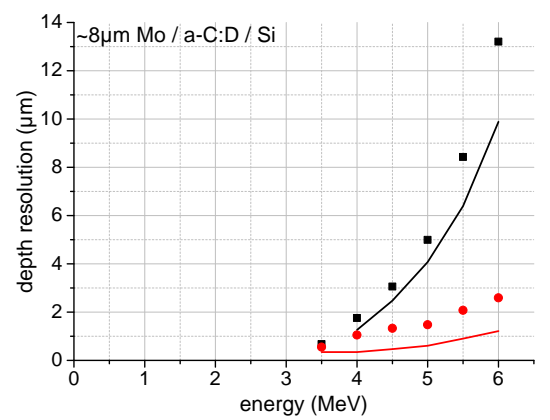

b)

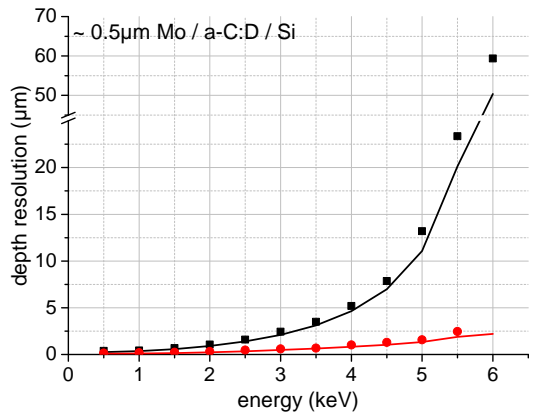

d)

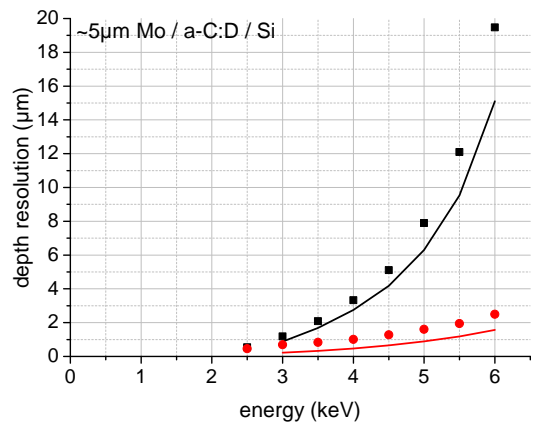

Figure 11: Depth resolution in $\mu \mathrm{m}$ versus incident ${ }^{3} \mathrm{He}$ energy in molybdenum for different thicknesses of the molybdenum cover layer. The dots are measured data. The lines are simulated data. The data from the annular detector at $175.9^{\circ}$ are in red. The data from the detector at $135^{\circ}$ are in black color in the figures. The depth resolution at the a-C:D / Si sample (figure 11a)) is calculated with the effective stopping power of an infinitesimally thin molybdenum cover layer. Please note the different axis scales at the figures and the axis breaks at some figures. In all above figures one can see that the better, i.e., lower, depth resolution is achieved with the detector at $175.9^{\circ}$. Comparing the depth resolution at high energies between experimental points an improvement up to a factor of 13 is reached. 
a)

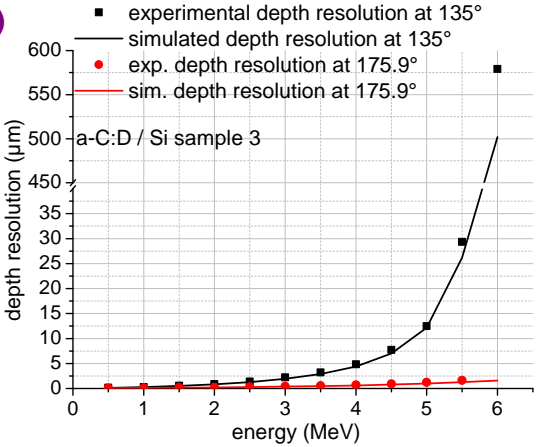

c)

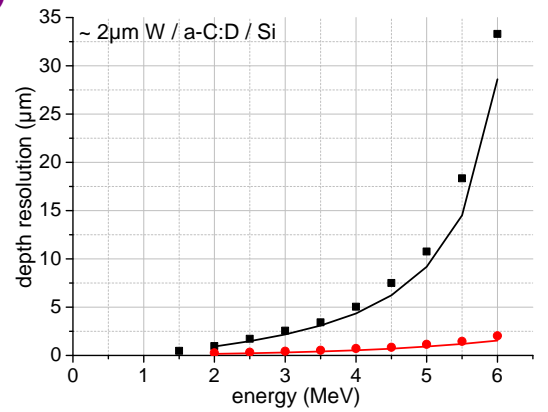

e)

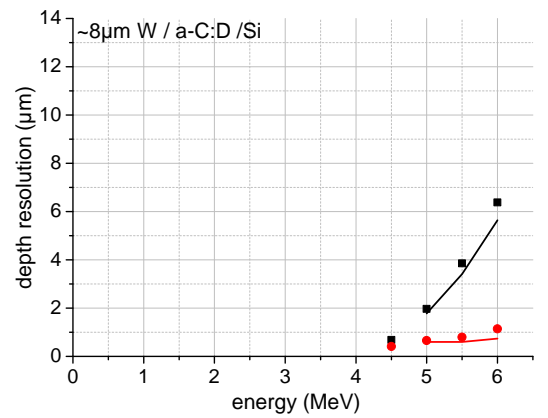

b)

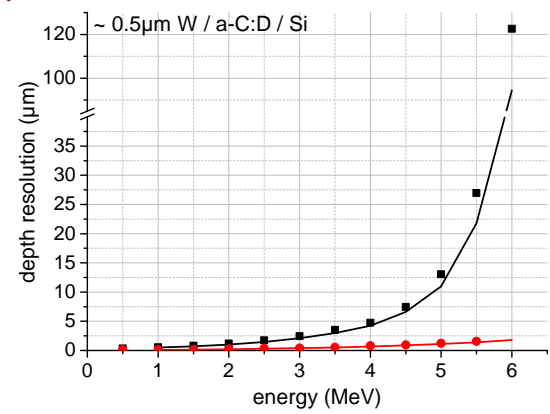

d)

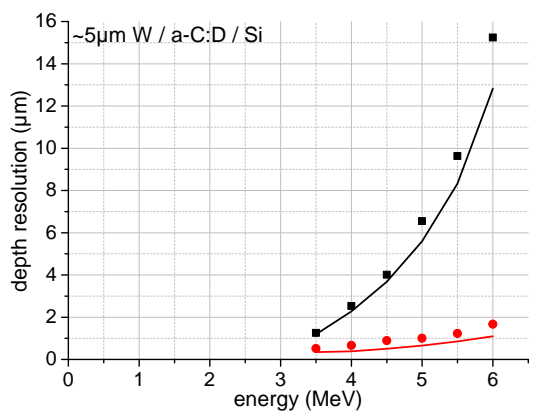

Figure 12: Depth resolution in $\mu \mathrm{m}$ versus incident ${ }^{3}$ He energy in tungsten for different thicknesses of the tungsten cover layer. The dots are measured data. The lines are simulated data. The data from the annular detector at $175.9^{\circ}$ are in red. The data from the detector at $135^{\circ}$ are in black color in the figures. The depth resolution at the a-C:D / Si sample (figure 12a)) is calculated with the effective stopping power of an infinitesimally thin tungsten cover layer. Please note the different axis scales at the figures and the axis breaks at some figures. In all above figures one can see that the better, i.e., lower, depth resolution is achieved with the detector at $175.9^{\circ}$. Comparing the depth resoltuion at high energies between experimental points an improvement up to a factor of 18 is reached. 


\section{Summary}

Kinematic considerations show that the best depth resolution for depth profiling with the $\mathrm{D}\left({ }^{3} \mathrm{He}, \mathrm{p}\right) \alpha$ reaction is achieved at angles of $0^{\circ}$ or $180^{\circ}$. In order to confirm this theoretical prediction the depth resolution of an annular proton detector at $175.9^{\circ}$ and a proton detector at $135^{\circ}$ were determined experimentally and compared with simulations.

For determination of the energy spread at different energies proton energy spectra from the $\mathrm{D}\left({ }^{3} \mathrm{He}, \mathrm{p}\right) \alpha$ nuclear reaction were recorded at reaction angles of $135^{\circ}$ and $175.9^{\circ}$. Different ${ }^{3} \mathrm{He}$ incident energies ranging from $0.5 \mathrm{MeV}$ to $6 \mathrm{MeV}$ with a step width of $0.5 \mathrm{MeV}$ were used.

To determine the energy spread in different materials and depths thin a-C:D layers on flat $\mathrm{Si}$ wafers buried under diverse cover metal layers (Al, Mo, W) were produced.

From the width of the proton peak in the spectra the energy spread was deduced. From this the depth resolution was calculated.

It could be shown that the depth resolution for the annular detector at $175.9^{\circ}$ is better for all materials and at all energies compared to the detector at $135^{\circ}$. The depth resolution was also simulated by SIMNRA and compared to the experimental depth resolution.

The trend of the experimental and simulated depth resolution with energy is very similar but the simulated depth resolution is somewhat lower at all energies and for all samples. This implies that one or more processes limiting the depth resolution are somewhat underestimated in the simulations.

\section{Acknowledgment}

The technical assistance with ion beam measurements by J. Dorner and M. Fußeder and scanning electron microscopy investigations by G. Matern are 
gratefully acknowledged.

[1] R. Langley, S. Picraux, F. Vook, Depth Distribution Profiling of Deuterium and ${ }^{3} \mathrm{He}$, Journal of Nuclear Materials 53 (1974) 257- 261.

[2] M. Mayer, E. Gauthier, K. Sugiyama, U. von Toussaint, Quantitative Depth Profiling of Deuterium up to very Large Depths, Nuclear Instruments and Methods in Physics Research Section B 267 (2009) 506-512.

[3] V. Alimov, M. Mayer, J. Roth, Differential Cross-Section of the $\mathrm{D}\left({ }^{3} \mathrm{He}, \mathrm{p}\right) \alpha$ Nuclear Reaction and Depth Profiling of Deuterium up to Large Depths, Nuclear Instruments and Methods in Physics Research Section B 234 (2005) 169.

[4] B. Wielunska, M. Mayer, T. Schwarz-Selinger, U. von Toussaint, J. Bauer, Cross section data for the $\mathrm{D}(3 \mathrm{He}, \mathrm{p}) 4 \mathrm{He}$ nuclear reaction from 0.25 to 6 $\mathrm{MeV}$, Nuclear Instruments and Methods in Physics Research Section B $371(2016) 41-45$.

[5] H. Bosch, G. Hale, Improved Formulas for Fusion Cross Sections and Thermal Reactivites, Nuclear Fusion 32 (1992) 111.

[6] M. Nocente, G. Gorini, J. Källne, M. Tardocchi, Cross Section of the $\mathrm{D}+{ }^{3} \mathrm{He} \longrightarrow \alpha+\mathrm{p}$ Reaction of Relevance for Fusion Plasma Applications, Nuclear Fusion 50 (5) (2010) 055001.

[7] M. H. J. 't Hoen, M. Mayer, A. W. Kleyn, P. A. Zeijlmans van Emmichoven, Strongly reduced penetration of atomic deuterium in radiation-damaged tungsten, Physical Review Letters 111 (2013) 225001.

[8] J. Roth, K. Schmid, Hydrogen in tungsten as plasma-facing material, Physica Scripta Volume T 145 (1) (2011) 014031. 
[9] M. Mayer, V. Rohde, G. Ramos, E. Vainonen-Ahlgren, J. Likonen, A. Herrmann, R. Neu, the ASDEX Upgrade Team, The deuterium inventory in ASDEX Upgrade, Nuclear Fusion 47 (11) (2007) 1607.

[10] J. Tesmer, M. Nastasi, Handbook of Modern Ion Beam Materials Analysis, MRS Material Research Society Pittsburgh Pennsylvania, 1995.

[11] E. Szilágyi, F. Pászti, G. Amsel, Theoretical approximations for depth resolution calculations in IBA methods, Nuclear Instruments and Methods in Physics Research Section B 100 (1995) 103.

[12] H. Ellmer, W. Fischer, A. Klose, D. Semrad, Assembly for Rutherford backscattering at exactly $180^{\circ}$, Review of Scientific Instruments 67 (5) (1996) 1794-1799.

[13] T. Schwarz-Selinger, A. von Keudell, W. Jacob, Plasma Chemical Vapor Deposition of Hydrocarbon Films: The Influence of Hydrocarbon Source Gas on the Film Properties, Journal of Applied Physics 86 (1999) 3988.

[14] M. Mayer, SIMNRA user's guide, Tech. Rep. IPP 9/113. Garching: MaxPlanck-Institut fur Plasmaphysik, 1997.

URL http://www.rzg.mpg.de/ mam/Report\%20IPP\%209-113.pdf

[15] I. Petrov, P. Barna, L. Hultmann, J. Greene, Microstrucutural Evolution during Film Growth, Vacuum Science and Technology A 21 (2003) 117. 